\title{
Les Simuliidae (Diptera) de trois rivières Oronte, Litani et Beyrouth du Liban
}

\author{
Z. Moubayed! \\ M. Clergue-Gazeau 1
}

Mots clès : Simuliidae, Liban, Zoogéographie.

Dans ce travail, les auteurs donnent une liste des Simuliidae recensées au Liban. Neuf espèces sont citées pour la première fois dans la région Est-méditerranéenne ; la présence du sous-genre Simulium s.s et de l'espèce Simulium (S.) variegatum s.l est à souligner. L'étude biogéographique du peuplement montre qu'il s'agit d'une faune paléarctique à distribution méditerranéenne assez large.

The Simuliidae (Diptera) of Lebanon (Rivers : Oronte, Litani and Beirut).

Keywords : Diptera, Simuliidae, Lebanon, Zoogeography,

In this paper, the authors list the Simuliidae recorded in Lebanon. Nine species are recorded for the first time in the East-mediterranean area ; the presence of Simulium s.s. subgenus and Simulitum (S.) variegatum s.l species has to be stressed. The biogeographical study of these blackflies reveals that we are dealing with palearctic fauna characterized by a widespread mediterranean distribution.

\section{1. - Introduction}

Les travaux de Crosskey (1967) et Dia (1983) constituent les seules données sur les Simuliidae du Liban. Nos recherches sur les invertébrés aquatiques du Liban nous ont permis d'obtenir de nouvelles données sur ce groupe.

\section{2. - Méthodes et localités d'étude}

Le matériel, composé de larves, nymphes, exuvies nymphales et imagos a été obtenu à partir de prélèvements benthiques avec un filet Surber (vide de maille $0,15 \mathrm{~mm})$ et de dérive $(0,15 \mathrm{~mm}$ ou $0,2 \mathrm{~mm})$. Une grande partie des adultes a été récoltée grâce aux chasses effectuées au filet entomologique, l'autre étant recueillie dans les filets de dérive.

1. Université Paul Sabatier, Laboratoire d'Hydrobiologie. U.A. 695 du C.N.R.S., 118, route de Narbonne, 31062 Toulouse Cédex, France.
Les campagnes de récoltes se sont échelonnées sur 3 années : juillet, août, septembre 1980 ; septembre, octobre 1981 ; mars, avril, mai 1982. Dans la mesure du possible nous les avons fait coïncider avec les périodes d'émergence des imagos.

Les différentes stations prospectées sont indiquées sur une carte du Liban (fig. 1): les stations 1 à 6 sont situées dans le bassin de la rivière Assi (= Oronte) ; 7 à 11 dans le bassin du Litani ; 12 et 13 dans le bassin du Beyrouth.

\section{1. - Bassin de l'Oronte}

- Station 1 : Oronte-Hermel, alt. $650 \mathrm{~m}$, hyporhithral (1a); avec méandres (1b), $\mathrm{t}^{\circ}$ $=\min 10 \cdot \max 15^{\circ} \mathrm{C}$.

- Station 2: Elaïn, alt. $950 \mathrm{~m}$, crénal avec ruisselet de source, $\mathrm{t}^{\circ}=9-12^{\circ} \mathrm{C}$.

- Station 3: Labwé, alt. $1000 \mathrm{~m}$, crénal avec ruisselets de sources, $t^{\circ}=9.12^{\circ} \mathrm{C}$. 


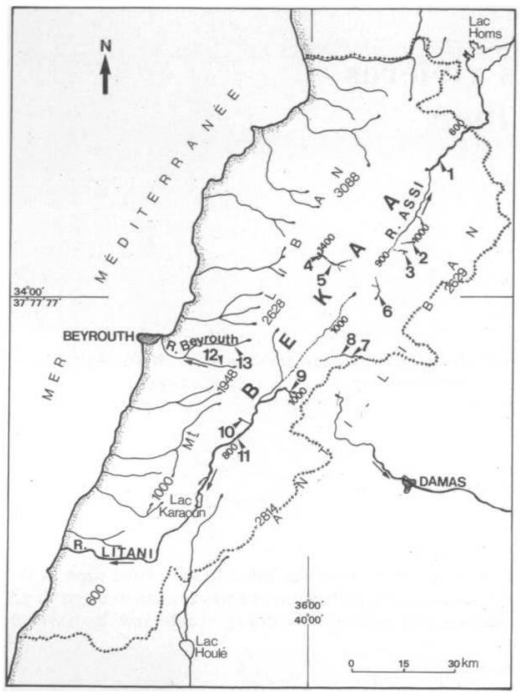

Fig. 1 Localisation des stations d'étude : 1 à 13.

- Station 4: Yammouné, alt. $1300-1400 \mathrm{~m}$, crénal $(4 \mathrm{a}$ et $4 \mathrm{~b}), \mathrm{t}^{\circ}=8-10^{\circ} \mathrm{C}$; épirhithral, métarhithral $(4 \mathrm{c}), \mathrm{t}^{\circ}=$ $6-12^{\circ} \mathrm{C}$.

- Station 5: Chlifa, alt. 1 100-1 $200 \mathrm{~m}$, métarhithral $(5 a$, courant rapide à très rapide), présence de mouilles et d'un replat $\left(5 b\right.$, courant lent), $t^{\circ}=$ $8,5-13^{\circ} \mathrm{C}$.

- Station 6: Baalbek, alt. $1100-1150 \mathrm{~m}$, crénal (6a, courant lent, $\mathrm{t}^{\circ}=9-12,5^{\circ} \mathrm{C}$ ), hyporhithral ( $6 \mathrm{~b}$, courant modéré), $\mathrm{t}^{\circ}=9-16^{\circ} \mathrm{C}$.

\section{2. - Bassin du Litani}

- Station 7: Yahfoufa, alt. $1200 \mathrm{~m}$, affluent du Litani, métarhithral, $\mathrm{t}^{\circ}=11-14^{\circ} \mathrm{C}$.

- Station 8: Janta, alt. $1100 \mathrm{~m}$, cours inférieur du Yahfoufa, hyporhithral (8a), $\mathrm{t}^{\circ}=$ $12.14^{\circ} \mathrm{C}$.
- Station 9: Anjar-Chamsine, alt. $900-1000 \mathrm{~m}$, affluent Ghozayel du Litani : crénal (9a), $t^{\circ}=14-18^{\circ} \mathrm{C}$, épipotamal supérieur $(9 \mathrm{~b}), \mathrm{t}^{\circ}=13.20^{\circ} \mathrm{C}$.

- Station 10: Ammik, alt. $850 \mathrm{~m}$, crénal (10a), $\mathrm{t}^{\circ}=1416^{\circ} \mathrm{C}$ et marais (10b).

- Station 11: Jib-Jennine, le Litani, alt. $800 \mathrm{~m}$, épipotamal inférieur pollué.

\section{3. - Bassin du Beyrouth}

- Stations 12 et 13 : Baalechmay:

- 12 : crénal, alt. $1000 \mathrm{~m}$;

- 13: hyporhithral, alt. $700 \mathrm{~m}$; courant rapide à modéré.

Pour plus de détails sur chaque station, se rapporter aux travaux de Moubayed et Laville (1983), Moubayed et Botosaneanu (1985).

\section{3. - Faunistique et répartition}

Nous donnons ci-dessous dans l'ordre de la classification de Crosskey (1981), la liste des espèces récoltées à l'état d'imago (Im), de nymphe (N) et de larve $(\mathrm{L})$ avec les localités de récoltes.

Simulium Eusimu-

lium) latizonum $\mathbf{R z} . \quad$ Im, N, L (13)

** S. (Nevermannia) ibleum Rz. 1

S. (Nevermannia)

sp. N. $(4 c ; 5 a)$

* S. (Odagmia) ornatum (Mg.) $)^{1}$

Im, N, L $(4 c ; 5 \mathrm{ab} ; 7 ; 8 \mathrm{a}$ : $10 \mathrm{a} ; 11)$

S. (O.) nitidifrons

Edw

$\mathrm{N}(13)$

*** S. (Simulium) variegatum $\mathrm{Mg} .{ }^{1}$

S. (Tetisimulium) bezzii Corti1

$\operatorname{Im}, \mathrm{N}, \mathrm{L}(3 ; 5 \mathrm{a} ; 7)$

Im, N, L $(1 a ; 3 ? ; 4 \mathrm{c} ; 7$; 13)

* S. (Wilhelmia) paraequinum (Puri) ${ }^{1}$ S. (W.) pseudequinum Séguy

$\operatorname{Im}, \mathrm{N},(1 \mathrm{ab})$

$\operatorname{Im}, \mathrm{N}(1 \mathrm{ab} ; 13)$

1. Ces espèces ont été identifiées par le $D^{r}$ R. Crosskey. 
** S. (W.) turgaicum

$$
\text { Rz. } 1 \text { Im, L (11) }
$$

S. (W.) ? veltis-

chevi (Rz.) N(13)

S. (W.) $s p . \quad \mathrm{N}(3 ; 6 \mathrm{~b} ; 7)$

*** espèce nouvelle pour le Moyen-Orient ;

** espèce nouvelle pour l'Est méditerranéen ;

* espece nouvelle pour le Liban.

\section{4. - Analyse du peuplement}

\section{1. - Considérations générales}

D'après Crosskey (1967) “ The composition of the Middle Eastern fauna is typical of that for the whole Mediterranean subregion of the Palearctic, from Morocco to west Pakistan, where the subgenera Wilhelmia and Eusimulium are dominant, Odagmia well represented and Simulium s. str. almost entirely absent... The subgenus Tetisimulium... is not yet known from the Middle East $»$. Dans notre matériel cinq espèces, soit près de la moitié du peuplement total, appartiennent au sous-genre Wilhelmia alors que les sous-genres Eusimulium et Odagmia ne sont respectivement représentés que par une ou deux espèces. La présence du sous-genre Simulium s. str. avec $S$. $(S$.) variegatum s.l est à souligner. Une autre espèce du même sous-genre: $S(S$.) reptans, figure vraisemblablement aussi dans nos échantillons en provenance de Yammouné (1 pupe et quelques larves) mais son identité demande à être confirmée. Enfin, nous mentionnerons l'absence, dans nos prélèvernents, de deux espèces qui normalement devraient y figurer, Prosimulium (P.) petrosum et $S$. (W.) golani. La première est signalée au Liban (Zahlé, Bcharré : rivières Damour et Awali) par Crosskey (1967) et Dia (1983). la seconde dans le massif du Golan (sud de l'Anti-Liban) par Beaucournu-Saguez \& al. (1976). Il es1 possible que les dates de nymphose et d'émergence de ces deux espèces ne correspondent pas à nos périodes de récoltes.

\section{2. - Répartition du peuplement}

\subsection{1. - Répartition au Líban}

Dia (1983) a dressé une liste de sept espèces ou taxa récoltés dans deux rivières côtières le Damour et l'Awali, du Liban méridional : $P$. (P.) petrostum, $S$. (E.) latizonum, S. (Obuchovia) cfr. gr. auricoma,
S. (O.) nitiditrons, $S$. (S.) gr. monticola n. $s p, S$. (T.) bezzii et $S$. (W.) pseudequinum. Quatre de ces espèces : $S$. (E.) latizonum, $S$. (O.) nitidifrons, $S$. (T.) bezzii et $S$. (W.) pseudequinum se retrouvent dans notre peuplement ; les deux premières sont récoltées uniquement dans le Beyrouth, quant aux deux autres. elles sont communes aux trois rivières.

Six espèces, $S$. (N.) ibleum, $S$. (O) ornatum, S. (S.) variegatum, S. (W.) paraequinum, S. (W.) turgaicum et $S$. (W.) ? veltischevi seraient nouvelles pour le Liban (les cinq premières ont été prélevées dans la Bekaa et la dernière dans le Beyrouth).

\subsection{2. - Répartition dans les localités d'étude}

Parmi les douze espèces identifiées, huit se rencontrent dans le bassin de l'Oronte et cinq dans chacun des bassins Litani et Beyrouth. La richesse spécifique maximale (cinq et quatre espèces) s'observe respectivement dans les stations : $\mathrm{S} 13$ (rivière Beyrouth) et S3, S4, S5 (rivière Oronte); notons trois espèces aux stations: $\mathbf{S 1}, \mathbf{S} 2, \mathbf{S} 11$ et une seule à $\mathbf{S 8}$ et $\mathrm{S} 10$.

\section{3. - Considérations écologiques}

Nous nous limiterons ici aux espèces obtenues à partir de prélèvements benthiques : $S$. (N.) ibleum, $S$. (O) ornatum, $S$. (S.) variegalum, $S$. (T.) bezzii, $S$. (W.) paraequinum et $S$. (W.) turgaicum. Nos observations sur les autres taxa, provenant de dérives, ne nous paraissent pas assez concluantes pour une étude écologique.

\section{- S. (N.) ibleum}

La plupart des espèces du sous-genre Nevermania sont communes dans les petits ruisseaux, les ruisselets ou les canaux d'irrigation (Crosskey 1967); des larves de $S$. (N.) ibleum ont été récoltées en Sicile $(600-700 \mathrm{~m})$ entre janvier et mars dans un ruisseau frais $\left(t^{\circ} 12^{\circ} \mathrm{C}\right.$ ), mais temporaire, parmi les rochers sans végétation (Rivosecchi 1978); elle est signalée par Crosskey et Ashford (1981) en Cyrénaique (Lybie) au mois de mars dans un petit ruisseau à fond pierreux mais avec végétation ; c'est une espèce du crénal et du rhithral (Zwick 1978).

$S$. (N.) ibleum se rencontre dans trois localités de la Békaa: Labwé (ruisselet de sources, $1000 \mathrm{~m}$ ), Yammouné (crénal et épirhithral, $1360 \mathrm{~m}-1330 \mathrm{~m}$ ) et Chlifa (métarhithral, $1200-1100 \mathrm{~m}$ ). Dans ces 
trois stations, les eaux sont constamment fraîches $\left(8,5-13^{\circ} \mathrm{C}\right)$, le fond rocheux ou pierreux présente des bryophytes en courant rapide à modéré ou des macrophytes en courant lent, dans les mouilles. En général, l'espèce forme des populations peu denses entre mars et mai, elle n'est jamais dominante dans les prélèvements. Espèces associées : $S$. (O.) ornatum, S. (T.) bezzii.

\section{- S. (O.) omatum}

D'après la littérature, $S$. (O.) ornatum est la forme la plus commune et la plus ubiquiste d'Europe : eurytherme, euryèce et polluo-résistante ; elle est bien connue pour son rôle dans la transmission de l'Onchocercose et la faculté des femelles de s'attaquer aux mammifères y compris l'homme en provoquant certains troubles.

Nous l'avons observée dans plusieurs habitats, du crénal au potamal : Yammouné, Chlifa, Yahfoufa, Janta, Ammik et Jib-Jennine. Les nymphes présentent une abondance maximale entre mars et avril à Yammouné ; constatation en accord avec Rubzov (1959-64) qui note de fortes densités entre février et avril. Dans le métarhithral de Yammouné (4c3) $S$. (T.) bezzii est présente dans les mêmes biotopes que $S$. (O.) omatum. Les deux espèces cohabitent mais leurs dates de nymphose sont décalées (aoûtseptembre pour $S$. (T.) bezzii). Autres espèces associées: S. (N.) ibleum, S. (S.) variegatum.

\section{- S. (S.) variegatum}

Elle se rencontre à des altitudes allant de 200 à $1600 \mathrm{~m}$ et apparaît comme une espèce rhéophile capable de supporter des courants violents à rapides (Davies 1966) ; c'est une espèce du rhithral et du potamal (Zwick 1978).

Dans la Békaa, $S$. variegatum colonise le cours supérieur de l'Oronte et du Litani : crénal de Labwé et métarhithral de Chlifa et de Yahfoufa. Dans ces trois stations, nous notons en particulier un substrat rocheux ou de pierres avec des bryophytes et un courant généralement rapide ou très rapide. Cette espèce ne figure dans nos prélèvements qu'avec de faibles effectifs, contrairement aux fortes densités constatées dans les torrents d'Europe Occidentale. Espèces associées : S. (O.) ornatum et S. (N.) ibleum.

\section{- S. (T.) bezzii}

Dans la littérature $S$. (T.) bezzii apparait comme une espèce d'eau courante à la fois eurytherme et résistante aux différentes formes d'eutrophisation. D'après Grenier (1953) les larves et les pupes se rencontrent en courant très rapide ou violent, entre 300 et $2000 \mathrm{~m}$, en compagnie de $S$. (S.) variegatum, $S$. (O.) auricoma et S. (S.) monticola ; d'après Zwick (1978), T. bezzii est un habitant du rhithral de ruisseaux de hautes montagnes à fort courant et sans macrophytes ; selon Rivosecchi (1978) elle vit dans le piémont de hautes montagnes en compagnie d'autres espèces méditerranéennes comme ornatum et aureum..., les femelles sont haematophages ; elles s'attaquent aux chevaux et au bétail surtout au niveau de l'oreille.

Au Liban S. (T) bezzit se rencontre aussi bien dans les rivières côtières que dans celles de la province in térieure ou Békaa. Elle est présente dans l'Oronte (S1, S3, S4), dans un affluent du Litani, le Yahfoufa (S7) et dans le Beyrouth (S13). Ses populations les plus denses s'observent à Yammouné où l'espèce cohabite avec S. (O.) ornatum; son maximum de nymphose, entre août et octobre, est en accord avec les résultats de Rubzov (1959-64). Autres espèces associées: $S$. (W.) pseudequinum.

\section{- S. (W.) paraequinum}

Selon Rubzov (l.c.) elle préfère les eaux fraîches et peut avoir plusieurs générations par an ; comme la plupart des espèces du sous-genre Wilhelmia les femelles sont de véritables haematophages en s'attaquant à la partie interne de l'oreille des chevaux (Wirtz 1983) et probablement des dromadaires (Crosskey 1967) ; elle se renconte du rhithral au potamal (Zwick 1978).

Au Liban, nymphes et imagos de $S$. (W.) paraequinum ont été récoltés uniquement dans l'hyporhith. ral de l'Oronte à Hermel (S1 : alt. $650 \mathrm{~m}$ ) où ses émergences, en accord avec Terterian (1957), s'étalent du printemps à l'automne. Des pontes ont été observées sur des feuilles immergées de Frênes et de Saules, mention déjà faite par Rubzov (l.c.), les ceufs sont déposés à I'intérieur du mésenchyme (15 à 20 pontes par feuille). $S$. (W.) paraequinum est une espèce fréquente dans l'hyporhithral de l'Oronte mais nous ne savons pas si elle colonise aussi d'autres sections de cette rivière. Espèces associées : $S$. (T.) bezzii et S. (W.) pseudequinum. 


\section{- (S.) (W.) turgaicum}

Les larves et les pupes de cette espèce prédominent dans les fossés et canaux d'irrigation qui dérivent de petits ou moyens cours d'eau, il y a plusieurs générations par an, trois au moins, les femelles sont haematophages (Rubzov 1959-64).

Au Liban $S$. (W.) turgaicum n'est présente que dans nos prélèvements en provenance de la Békaa (Litani à Jib-Jennine, $\mathrm{S} 1 \mathrm{I}$ ). Les récoltes d'adultes dans cette station s'étalent de mars à juin, sa répartition dans cette plaine s'étendrait aux nombreux canaux d'irrigation issus du Litani. C'est une espèce peu abondante dans l'épipotamal du Litani où elle se trouve associée parfois à $S$. $(O$.) ornatum.

\section{4. - Considérations biogéographiques et conclusion}

La faune de Simuliidae actuellement connue du Liban se compose de 11 espèces : 10 citées dans ce travail auxquelles s'ajoute $P$. (P.) petrosum signalée par Crosskey (1967) et par Dia (1983). La présence de $S$. (S.) reptans au Liban (Yammouné) demande confirmation.

Neuf espèces : $S$. (E.) latizonum, $S$. (N.) ibleum, $S$. (O.) nitidifrons, S. (O.) ornatum, S. (S.) variegatum, $S$. (T.) bezzii, S. (W.) turgaicum et $S$. (W.)? veltischevi sont nouvelles pour la Méditerranée orientale. Soulignons, aussi, l'extension à cette région du sousgenre Simulium s. str.

En comparaison avec les autres peuplements des régions limitrophes, cinq espèces sont communes avec le peuplement d'Afrique du Nord, six à huit avec la faune balkanique, sept avec celle du Caucase et d'Asie Mineure et enfin trois à quatre avec celle d'Israël. Ces résultats, tout à fait en accord avec l'opinion de Crosskey (1967), nous permettent de constater qu'il s'agit bien d'espèces paléarctiques à répartition méditerranéenne assez large.

Toutefois, la présence au Liban de trois ou quatre espèces, encore inconnues du reste du pourtour méditerranéen, témoigne d'une certaine originalité du fait des conditions climatiques ( 1 à 3 mois de gel) et du régime thermique des eaux $\left(8,5-18^{\circ} \mathrm{C}\right)$ qui prédominent principalement dans les bassins supérieurs de l'Oronte et du Litani (Békaa).
Etudiant la biogéographie des Trichoptères de la province levantine septentrionale, Moubayed et Botosaneanu (1985) séparent cette région en trois zones : zone I = bassins supérieurs de l'Oronte et du Litani ; zone II = rivières còtières libanaises ; zone III = bassin supérieur du Jourdain (Lac Kinnereth y compris).

En ce qui concerne le groupe des Simulies, nous pouvons opposer le peuplement de la zone I à celui de la zone II, deux zones séparées l'une de l'aut re par la chaîne côtière du Mont-Liban (alt. $3088-1000 \mathrm{~m}$ ).

- Zone I; versant est du Mont-Liban : $P$. (P.) petrosum, $S$. (N.) ibleum, $S$. (O) ornatum, $S$. (S.) variegatum, S. (T.) bezzii, S. (W.) paraequinum, S. (W.) pseudequinum, $S$. (W.) turgaicum; elle comporte au moins deux espèces asiatiques.

- Zone II ; versant ouest du Mont-Liban : $P$. (P.) petrosum, $S$. (E.) latizonum, $S$. (O) nitidifrons, S. (T.) bezzii, $S$. (W.) pseudequinum; représentée surtout par des espèces circum-méditerranéennes.

Trois espèces se trouvent communes aux deux zones: $P$. (P.) petrosum, $S$. (T.) bezzii et $S$. (W.) pseudequinum.

Notre étude a souligné l'extension géographique de trois espèces particulièrement intéressantes:

a - S. (N.) ibleum, qui selon Crosskey \& Ashford (1981) est probablement assez répandue dans le centre et l'Est de la région méditerranéenne (Sicile, îles grecques, Lybie); sa présence au Liban (zone I) confirme, par conséquent, cette hypothèse.

b - S. (W.) paraequinum présente dans le massif du Golan - plateau sud de l'Anti-Liban (Beaucournu-Saguez \& al. 1976) et au Liban (Békaa). La province levantine constitue bien la limite occidentale de son aire de distribution comme l'a constaté Crosskey (1967).

c - S. (W.) turgaicum est une espèce largement répandue en Asie mineure (Rubzov 1959-64; Crosskey 1967); sa présence au Liban peut être considérée comme la citation à la fois la plus méridionale et la plus occidentale.

\section{Remerciements}

Nous remercions cordialement le Dr. H. Zwick (Schlitz) pour sa précieuse contribution à ce travail et le $\mathrm{Dr}, \mathrm{R}$. Crosskey (Londres) pour l'identification d'une partie du matériel. 


\section{Travaux cités}

Beaucournu-Saguez (F.), Braverman (Y.) \& Tsafir (N.). 1976. - A new black-fly S. (W.) golani $n$. $s p$ (Diptera, Simuliidae) from the eastern Mediterranean basin. Bull. Soc. Pathol. Exot. Paris, 69 (3) : 272-278.

Crosskey (R.W.). 1967. - A preliminary revision of the black-flies (Diptera : Simuliidae) of the Middle East. Trans. $R$. ent. Soc. Lond. 119 (1): 1.45 .

Crosskey (R.W.). 1981. - Simuliid Taxonomy - The contemporary Scene - Blackflies : 3-18 in The future for biological methods in integrated control. Laird M., ed. Academic Press. London. $399 \mathrm{p}$.

Crooskey (R.W.) \& Ashford (R.W.). 1981. - The occurence of Simulium $\mathbf{s}$. I. in the Libyan Arab Republic. Annis Trop. Med. Parasitol. Aiverpool), 75 (6) : 047-651.

Davies (L.). 1966. - The taxonomy of British black-flies (Diptera : Simuliidae). Trans. R. ent. Soc. Lond. 118 (14) : 413.511.

Dia (A.). 1983. - Recherches sur l'écologie et la biogéographie des cours d'eau du Liban meridional. Thèse d'Etat, Université AixMarseille III ; $302 \mathrm{p}$.
Grenier (P.). 1953. - Simulidae de France et d'Afrique du Nord. Encyclop. Entomol., Ser. A, 29 : 1-170.

Moubayed (Z.) \& Laville (H.). 1983. - Les Chironomides (Diptera) du Liban. I. Premier inventaire faunistique. Annts Limnol., 19 (3) : 219.228 .

Moubayed (Z.) \& Botosaneanu (L.). 1985. - Recherche sur les Trichopteres du Liban et principalement des bassins superieurs de I'Oronte et du Litani. (Insecta : Trichoptera). Bull Zool Mus. Amsterdam (sous presse).

Rivusecchi (L.) 1978. - Simulidae Diptera Nematocera. Fauna d'Italia vol. XIII. Edizioni Calderini Bologna 533 p.

Rubzov (I.A.). 1959-64 - Simulitdae (Melusinidae). In Lindner (E.) ed. Die Fliegen der Paläarktischen Region. Bd. III (4) : 1.689.

Terterian (A.E.). 1957. - The determination of the number of instars in the larvae of black-flies (Diptera, Simuliidae). Ent. Obozr. $36: 860-868$.

Wirtz (P.). 1983. - Nahrungsaufnahme und Natalität bei palaearktischen und afrikanischen Simuliiden (Diptera). Diss. Fak. Biol. Univ. Tübingen, $102 \mathrm{p}$

Zwick (H.). 1978. - Simuliidae (Diptera). In Illies J. ed. Limnotauna Europaca : $396-403$ 